\title{
Osteopontin and vasculogenic mimicry formation are associated with response to neoadjuvant chemotherapy in advanced breast cancer
}

This article was published in the following Dove Press journal:

OncoTargets and Therapy

18 August 2017

Number of times this article has been viewed

Ming $\mathrm{Gu}^{1,2}$

Xinyu Zheng ${ }^{1,2}$

'Department of Breast Surgery, ${ }^{2}$ Department of Surgical Oncology, Research Unit of General Surgery, The First Affiliated Hospital of China Medical University, Shenyang, Liaoning, People's Republic of China
Correspondence: Xinyu Zheng Department of Breast Surgery, Research Unit of General Surgery, The First Affiliated Hospital of China Medical University, 155 Nanjing North Street, Heping District, Shenyang, Liaoning I I000I, People's Republic of China Tel/fax +86248328 274l

Email xinyu66II88@I26.com
Introduction: Osteopontin (OPN), a multifunctional phosphoprotein, has been implicated in a series of important physiologic and pathophysiologic processes. In breast cancer, OPN functionally contributes to the tumorigenicity of spheroid-forming cells. It also plays a critical role in enhancing the proliferation, tumorigenicity, and ability to display vasculogenic mimicry (VM) of spheroid-forming cells in breast cancer. However, the role of OPN in breast cancer is not clear.

Patients and methods: This study investigated OPN expression and VM in breast cancer patients before neoadjuvant chemotherapy (NACT). Their association with clinicopathologic factors was first analyzed by immunohistochemistry. Then, the response of breast cancer patients to NACT was evaluated. The correlation between the clinicopathologic factors, including the molecular subtype, and the response to NACT was analyzed.

Results: Immunohistochemical analysis showed positive staining of OPN in $40 \%$ of the breast cancer patients, whereas VM, which was related to tumor stage, was observed in $30 \%$ of cases. OPN expression was found to have a significant correlation with $\operatorname{VM}(P<0.05)$. The results also indicated that the clinicopathologic factors were not related to the response to NACT, including the molecular subtype. The multivariate analysis of clinicopathologic features correlated with pathological complete response $(\mathrm{pCR})$ indicated that $\mathrm{OPN}(+) \mathrm{VM}(+)$ was correlated with pCR $(P<0.001)$.

Conclusion: Our findings underlined that the concurrence of OPN-positive expression and VM can predict the pCR to NACT in breast cancer. The efficiency of NACT in certain patients can be easily predicted by detecting the expression of OPN and VM.

Keywords: osteopontin, vasculogenic mimicry formation, breast cancer, neoadjuvant chemotherapy

\section{Introduction}

Osteopontin (OPN), a multifunctional phosphoprotein, is involved in a series of important physiologic and pathophysiologic processes, including host defense, wound healing, and bone formation, by stimulating macrophage migration, as well as by protecting against viral and bacterial infections through its pro-Th1 effect. ${ }^{1-6}$ OPN may play multiple roles in promoting carcinogenesis and tumor progression in various cancers, including breast cancer, stomach cancer, colorectal cancer, lung cancer, ovarian cancer, esophageal cancer, and melanoma. ${ }^{7-18}$ A previous study indicated that OPN was a key mediator of vasculogenic mimicry (VM) in hepatocellular carcinoma and was correlated with poor prognosis. ${ }^{19}$ In breast cancer, OPN functionally contributes to the tumorigenicity of spheroid-forming cells. It plays a critical role in enhancing the 
proliferation, tumorigenicity, and ability to display VM of spheroid-forming cells in breast cancer. ${ }^{20,21}$

Neoadjuvant chemotherapy (NACT) initially used in breast cancer patients with inoperable locally advanced tumors or in cases with initially operable tumors is superior for increasing the chance of achieving breast-conserving surgery, evaluating the susceptibility of chemotherapy drugs, and assessing the response to chemotherapy. ${ }^{22}$ Patients with pathological complete response (pCR) after NACT have better chances of disease-free survival. ${ }^{23}$ However, the relation between OPN, VM, and response to NACT in breast cancer is not clear.

\section{Patients and methods}

\section{Clinical data}

A total of 200 patients with biopsy-proven breast cancer were recruited from the Department of Breast Surgical, The First Affiliated Hospital of China Medical University (China), between June 2006 and December 2014. The patients' ages ranged from 23 to 76 years, with an average age of 45.5 years. Each case was reviewed independently by two pathologists with a subspecialty focus in breast pathology, and only those cases in which both pathologists reached a unanimous final diagnosis were included. The pathology classification was according to the World Health Organization (WHO) criteria published by Tavassoli et al. ${ }^{24}$ The study was approved by the regional ethics committee of China Medical University. Written informed consent for the acquisition of tissue samples and medical records was obtained from all patients.

\section{NACT regimens}

The NACT regimens were given as follows: CEF $\left(600 \mathrm{mg} / \mathrm{m}^{2}\right.$ cyclophosphamide, $80 \mathrm{mg} / \mathrm{m}^{2}$ epirubicin, and $600 \mathrm{mg} / \mathrm{m}^{2}$ 5 -fluorouracil) every 3 weeks, EC $\left(600 \mathrm{mg} / \mathrm{m}^{2}\right.$ cyclophosphamide and $80 \mathrm{mg} / \mathrm{m}^{2}$ epirubicin) every 3 weeks, and TC (75 mg/m $\mathrm{m}^{2}$ docetaxel and $600 \mathrm{mg} / \mathrm{m}^{2}$ cyclophosphamide) every 3 weeks. The patients received NACT for a median of six cycles (range four to eight cycles) before surgery.

\section{Evaluation of NACT response}

After the NACT, all patients underwent the modified radical mastectomy or conservative surgery. The therapy response was assessed according to the Response Evaluation Criteria in Solid Tumors (RECIST). The clinical response was categorized into four groups: complete response (CR) was defined as complete resolution of all tumors as determined by physical examination and imaging studies, partial response (Pr) was defined as an incomplete reduction of $>50 \%$ in tumor size, stable disease (SD) was defined as a reduction of $<50 \%$ in tumor size, and progressive disease (PD) was defined as an increase in tumor size. The pathologic size was defined as the greatest dimension of residual invasive tumor and was categorized by using the revised tumor-node-metastasis (TNM) staging system of the American Joint Committee on Cancer. ${ }^{24}$ The side reactions in each group were recorded. Before and after NACT in each group, biopsy specimens of the breast cancer were collected from all cases. The sections of each specimen were stained with hematoxylin and eosin (H\&E) and observed at $200 \times$ magnification. A high-power field with epithelium was randomly selected from each section. Pathologic analysis on the surgical resection margins was also done in all cases.

\section{Immunohistochemical staining}

Formalin-fixed, paraffin-embedded specimens were cut into $4 \mu \mathrm{m}$-thick sequential sections. These sections were dewaxed in xylene, rehydrated stepwise in ethanol, and then boiled in citrate buffer ( $\mathrm{pH}$ 6.0) for 90 seconds within an autoclave. Endogenous peroxidase activity and nonspecific binding were blocked with $3 \% \mathrm{H}_{2} \mathrm{O}_{2}$ and nonimmune sera, respectively. The sections were then incubated with primary mouse anti-human OPN monoclonal antibody LFMb-14 (sc-73631; Santa Cruz Biotechnology, Inc., CA, USA) at 1:400 dilution overnight at $4^{\circ} \mathrm{C}$. Thereafter, a catalyzed signal amplification system (Maixin Biotechnology, Fuzhou, Fujian, China) was used for OPN staining according to the manufacturer's instructions. For the negative control, phosphate-buffered saline (PBS) was used instead of primary antibodies. The German semiquantitative scoring system was applied in combination with the staining intensity and area extent, which have been widely accepted and used in previous studies. ${ }^{20-22,25-27}$ Each lesion was scored according to the intensity of the nucleic staining (no staining $=0$, weak staining $=1$, moderate staining $=2$, and strong staining $=3)$ and the extent of stained cells $(0 \%=0$, $1 \%-10 \%=1,11 \%-50 \%=2,51 \%-80 \%=3$, and $81 \%-100 \%=4$; negative means $0 \%$ area staining, focally positive means $1 \%-80 \%$ area staining, and diffusely and positive means $81 \%-100 \%$ area staining). The final immunoreactive score was determined by multiplying the intensity scores with the extent of positivity scores of the stained cells, with a minimum score of 0 and a maximum score of $12 .{ }^{20,21,25,26}$ The slides were independently examined by the two pathologists previously mentioned. If there was a discrepancy between individual scores, both pathologists reevaluated the scores together until a consensus was reached before combining the individual scores. To obtain the statistical results, a final score of $\leq 1$ was considered as negative, whereas a score of $\geq 2$ was considered as positive. A scoring of OPN 
staining portion $(+++,++,+,-)$ was used to establish an OPN/VM correlation. A proportion score that represented the estimated proportion of positively stained tumor cells (negative, none;,$+<10 \% ;++, \geq 10 \%-<50 \% ;+++, \geq 50 \%$ ) was evaluated.

\section{Cluster of differentiation 3I (CD3I)- periodic acid-Schiff (PAS) dual staining}

Immunostaining was carried out as previously described. Anti-CD31 antibodies (Beijing Zhongshan Biotechnology Ltd Co., Beijing, China) were applied to the sections at a dilution ratio of 1:100. Later, the sections immunohistochemically stained with CD31 were further stained with PAS, followed by counterstaining with hematoxylin. These double-stained sections were used to observe the microvascular and VM structures. Quantification of VM was done by counting the VM channels and endothelium-dependent vessels in the H\&E-stained sections under a microscope at 400× magnification; the CD31-PAS dual-stained sections were viewed at $400 \times$ magnification. The channels defined as VM were lined with PAS-positive material, with red cells in the center of the channels, but not with CD31-positive endothelial cells. ${ }^{28}$
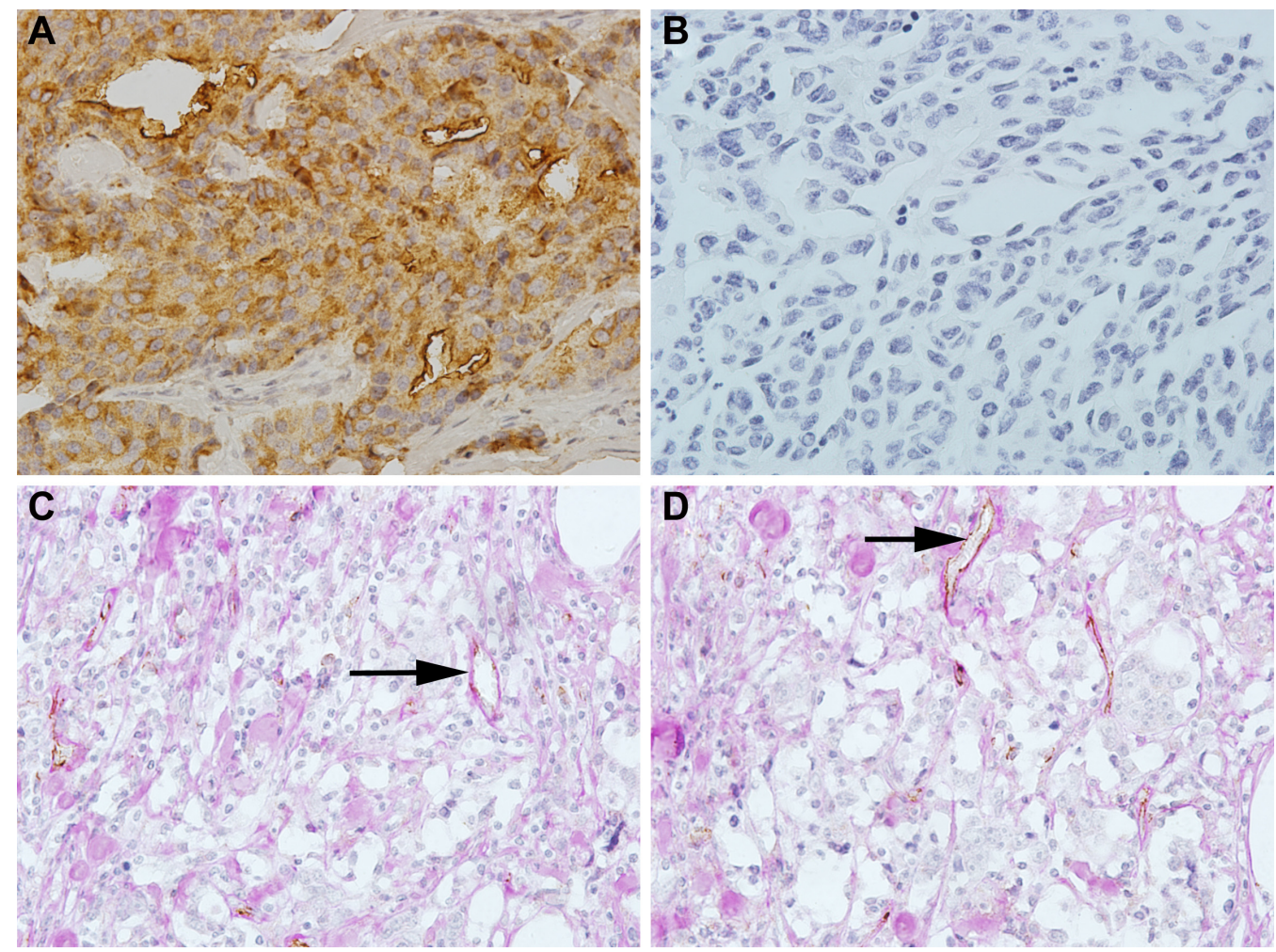

Figure I Representative immunohistochemical staining of OPN in breast cancer.

Notes: (A) Positive OPN immunostaining in invasive breast cancer; the OPN expression patterns were cytoplasmic staining. (B) Negative immunostaining of OPN in breast cancer. (C) VM in breast cancer tissue (black arrow, VM channels formed by tumor cells). (D) Typical blood vessels with brown CD3 ${ }^{+}$staining (black arrow). Original magnification, 200x.

Abbreviations: OPN, osteopontin; VM, vasculogenic mimicry. 
Table I Expression of OPN in 200 patients with breast carcinoma before NACT

\begin{tabular}{|c|c|c|c|c|}
\hline \multirow[t]{2}{*}{ Characteristics } & \multirow[t]{2}{*}{$\mathbf{N}$} & \multicolumn{2}{|c|}{ Expression of OPN } & \multirow[t]{2}{*}{$P$-value } \\
\hline & & $\begin{array}{l}\begin{array}{l}\text { Positive } \\
(\mathrm{n}=\mathbf{8 0})\end{array} \\
\end{array}$ & $\begin{array}{l}\text { Negative } \\
(n=\mid 20)\end{array}$ & \\
\hline \multirow[t]{2}{*}{ Age (years) } & & & & $\chi^{2}=1.47$ \\
\hline & & & & $P>0.05$ \\
\hline$<45$ & 98 & 35 & 58 & \\
\hline$\geq 45$ & 102 & 45 & 57 & \\
\hline \multirow[t]{2}{*}{ Menopausal status } & & & & $\chi^{2}=1.35$ \\
\hline & & & & $P>0.05$ \\
\hline Premenopausal & 90 & 32 & 58 & \\
\hline Postmenopausal & 110 & 48 & 62 & \\
\hline \multirow[t]{2}{*}{ Tumor size $(\mathrm{cm})$} & & & & $\chi^{2}=0.00$ \\
\hline & & & & $P>0.05$ \\
\hline$\leq 2$ & 9 & 3 & 6 & \\
\hline$>2$ & 191 & 77 & 114 & \\
\hline
\end{tabular}

Abbreviations: OPN, osteopontin; NACT, neoadjuvant chemotherapy.

cytoplasm of breast cancer cells. Positive staining of OPN was seen in $80(40 \%)$ of 200 specimens of breast cancer. OPN expression was not related to age, tumor size, and menopausal status (Table 1).

\section{$\mathrm{VM}$ in breast cancer specimens before NACT}

Staining of the endothelial cell marker CD31 was used to detect the endothelium in cancer tissue sections, whereas PAS staining was used to determine the basement membrane of tumor blood vessels. CD31-negative, PAS-positive, and vascular-like patterns containing red blood cells were identified as VM positive. VM was observed in 30\% (60 of 200) of the breast cancer samples. VM was not related to age, tumor size, lymph node metastasis, and menopausal status but was associated with tumor stage (Table 2).

\section{Correlation between OPN expression and $\mathrm{VM}$ in breast cancer}

Our results indicated that OPN expression had a significant correlation with VM $(P<0.01)$, as shown in Table 3.

\section{Response to NACT}

All patients were found to be eligible for modified radical mastectomy or conservative surgery after the treatment. The 200 breast cancer patients were evaluable for response to NACT, with an overall pCR rate of $41 \%$ $(n=200)$ for the entire cohort. Tables 4 and 5 list the clinical and pathologic response rates to NACT, respectively. Figure 2 presents a breast cancer case who obtained pCR after NACT. No correlation was found between the
Table 2 VM in 200 patients with breast carcinoma before NACT

\begin{tabular}{|c|c|c|c|c|}
\hline \multirow[t]{2}{*}{ Characteristics } & \multirow[t]{2}{*}{$\mathbf{N}$} & \multicolumn{2}{|l|}{ VM } & \multirow[t]{2}{*}{$P$-value } \\
\hline & & $\begin{array}{l}\text { Positive } \\
(n=60)\end{array}$ & $\begin{array}{l}\text { Negative } \\
(n=\mid 40)\end{array}$ & \\
\hline \multirow[t]{2}{*}{ Age (years) } & & & & $\chi^{2}=0.03$ \\
\hline & & & & $P>0.05$ \\
\hline$<45$ & 98 & 30 & 68 & \\
\hline$\geq 45$ & 102 & 30 & 72 & \\
\hline \multirow[t]{2}{*}{ Menopausal status } & & & & $\chi^{2}=0.10$ \\
\hline & & & & $P>0.05$ \\
\hline Premenopausal & 90 & 26 & 64 & \\
\hline Postmenopausal & 110 & 34 & 76 & \\
\hline \multirow[t]{2}{*}{ Tumor size $(\mathrm{cm})$} & & & & $\chi^{2}=0.02$ \\
\hline & & & & $P>0.05$ \\
\hline$\leq 2$ & 9 & 3 & 6 & \\
\hline$>2$ & 191 & 57 & 134 & \\
\hline
\end{tabular}

Abbreviations: VM, vasculogenic mimicry; NACT, neoadjuvant chemotherapy.

Table 3 Correlation of OPN expression and VM in breast cancer

\begin{tabular}{llll}
\hline & VM & & P-value \\
\cline { 2 - 3 } & Positive & Negative & \\
\hline OPN expression +++ & 29 & 20 & $\begin{array}{r}r=0.508 \\
P<0.001\end{array}$ \\
OPN expression ++ & 17 & 10 & \\
OPN expression + & 2 & 12 & \\
OPN expression - & 12 & 108 & \\
\hline
\end{tabular}

Abbreviations: OPN, osteopontin; VM, vasculogenic mimicry.

Table 4 Clinical and pathologic response rates to NACT

\begin{tabular}{ll}
\hline Pathologic response & Breast cancer $(\mathbf{n}=\mathbf{2 0 0})$ \\
\hline PCR, $n(\%)$ & $41(20.5)$ \\
Non-pCR, $n(\%)$ & $159(79.5)$ \\
Pr, $n(\%)$ & $148(74.0)$ \\
SD, $n(\%)$ & $15(7.5)$ \\
PD, $n(\%)$ & $9(4.5)$ \\
RR $(C R+P r), n(\%)$ & $176(88.0)$ \\
\hline
\end{tabular}

Abbreviations: NACT, neoadjuvant chemotherapy; $\mathrm{PCR}$, pathological complete response; $\mathrm{Pr}$, partial response; SD, stable disease; PD, progressive disease; RR, response rate; $\mathrm{CR}$, complete response.

Table 5 Logistic regression analysis of clinicopathologic features correlated with $\mathrm{PCR}$

\begin{tabular}{|c|c|c|c|c|c|}
\hline \multirow[t]{2}{*}{ Factor } & \multicolumn{2}{|c|}{ PCR } & \multirow{2}{*}{$\begin{array}{l}\text { Odds } \\
\text { ratio }\end{array}$} & \multirow{2}{*}{$\begin{array}{l}95 \% \\
\text { confidence } \\
\text { interval }\end{array}$} & \multirow[t]{2}{*}{$P$-value } \\
\hline & + & - & & & \\
\hline OPN & 21 & 59 & 1.780 & 0.89 I, 3.554 & 0.102 \\
\hline $\mathrm{VM}$ & 17 & 43 & 1.911 & $0.937,3.899$ & 0.075 \\
\hline ER & 25 & 94 & 1.080 & $0.535,2.182$ & 0.829 \\
\hline PR & 21 & 84 & 0.938 & $0.472,1.863$ & 0.854 \\
\hline HER2 & 13 & 50 & 1.012 & $0.484,2.117$ & 0.974 \\
\hline Age & 22 & 76 & 1.265 & $0.635,2.517$ & 0.504 \\
\hline Tumor size & 3 & 6 & 2.013 & $0.481,8.418$ & 0.338 \\
\hline Menopausal status & 16 & 74 & 0.735 & $0.365, I .48 \mathrm{I}$ & 0.389 \\
\hline
\end{tabular}

Abbreviations: ER, estrogen receptor; $P C R$, pathological complete response; $\mathrm{PR}$, progesterone receptor; OPN, osteopontin; VM, vasculogenic mimicry. 

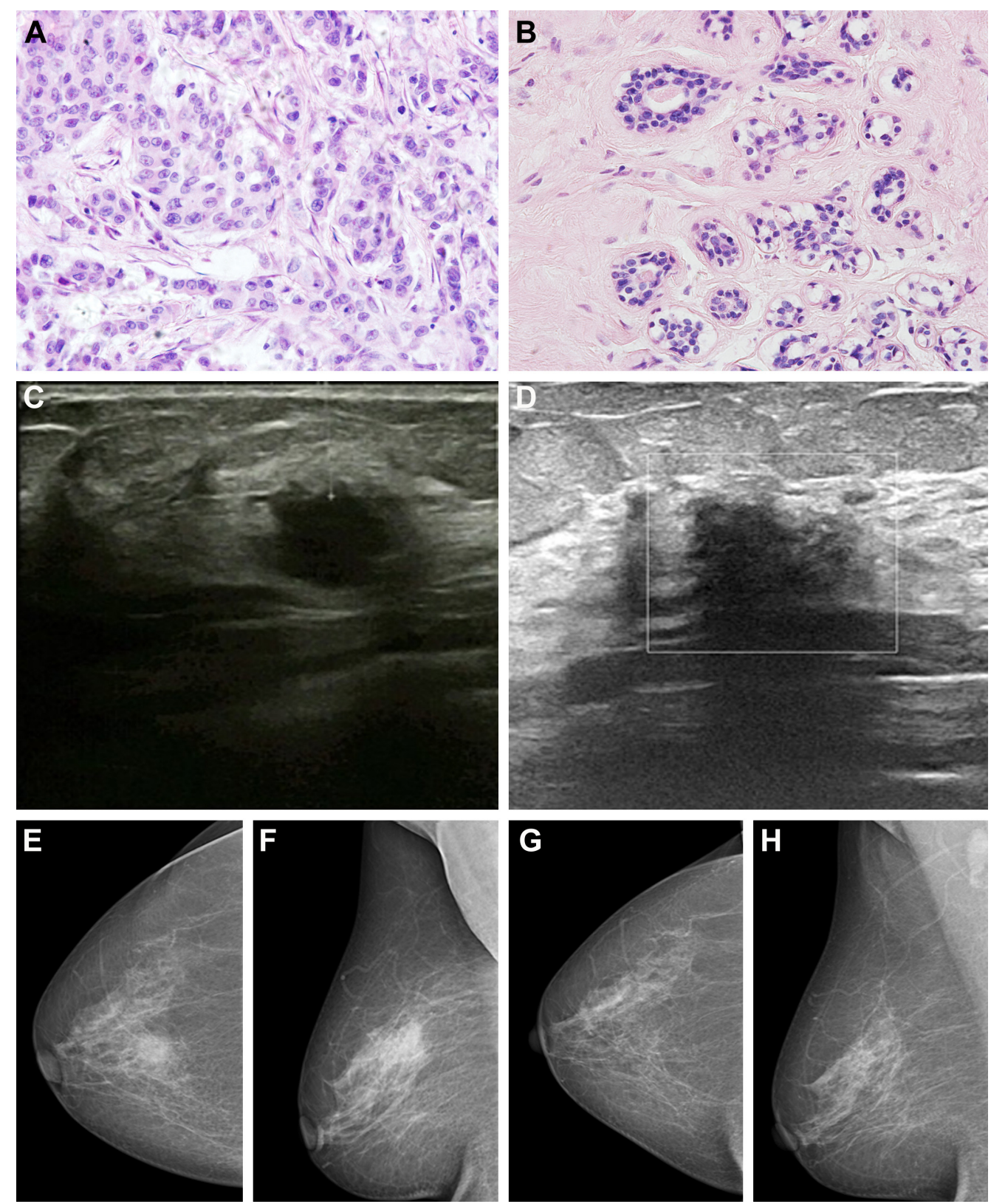

Figure 2 The difference between before and after NACT.

Notes: Pathologic images of breast cancer before (A) and after (B) NACT. Sonographic volume measurement of breast cancer mass before (C) and after (D) NACT. Mammography of breast cancer mass before (E and $\mathbf{F})$ and after ( $\mathbf{G}$ and $\mathbf{H})$ NACT.

Abbreviation: NACT, neoadjuvant chemotherapy.

clinicopathologic factors and the response to NACT, including the molecular subtype.

\section{Multivariate analysis of clinicopathologic features correlated with PCR}

In the multivariate analysis of clinicopathologic features correlated with pCR, OPN expression, VM, and other clinicopathologic features, such as tumor size and ER expression, showed no statistically significant correlation, as presented in Table 6. OPN $(+) \mathrm{VM}(+)$ is shown to be correlated with pCR $(P<0.001)$.

\section{Discussion}

Breast cancer is the leading cause of cancer death in women worldwide, with a high morbidity and mortality, especially in China. OPN is a chemokine-like protein that is involved in the promotion of neoplastic cancer into higher grade malignancies by regulating various facets of tumor progression, such as cell proliferation, angiogenesis, and metastasis. ${ }^{29}$ A previous study indicated that OPN was required for VM in hepatocellular carcinoma and was associated with activation of MMP-2 and uPA. ${ }^{19}$ OPN functionally contributes to the 
Table 6 Correlation between pCR, OPN, and VM

\begin{tabular}{|c|c|c|c|c|}
\hline & \multicolumn{2}{|c|}{ PCR } & \multirow[t]{2}{*}{$\chi^{2}$} & \multirow[t]{2}{*}{$P$-value } \\
\hline & + & - & & \\
\hline OPN(+)VM(+) & 15 & 27 & 7.551 & 0.006 \\
\hline OPN(+)VM(-) & 6 & 32 & 0.639 & 0.424 \\
\hline OPN(-)VM(+) & 2 & 16 & 0.377 & 1.070 \\
\hline OPN(-)VM(-) & 18 & 84 & 1.040 & 0.308 \\
\hline
\end{tabular}

Abbreviations: pCR, pathological complete response; OPN, osteopontin; VM, vasculogenic mimicry.

tumorigenicity of spheroid-forming cells in breast cancer. It plays a critical role in enhancing the proliferation, tumorigenicity, and ability to display VM of spheroid-forming cells in breast cancer. ${ }^{20,21,26} \mathrm{NACT}$ is a conventional preoperative treatment for breast cancer. ${ }^{30}$ The pCR to NACT can help predict long-term survival in patients with breast cancer, and the pCR definition most strongly correlated with improvement in survival outcomes. ${ }^{31}$ Thus, the pCR to NACT should be encouraged.

The objective of this study was to assess the relation between OPN, VM, and response to NACT in breast cancer. Our results indicated that OPN was expressed in $40 \%$ of the breast cancer patients, whereas VM was observed in 30\% of cases. OPN expression showed a significant correlation with $\mathrm{VM}$ in the breast cancer samples $(P<0.05)$. However, in the multivariate analysis of clinicopathologic features correlated with pCR, OPN expression and VM showed no statistically significant correlation. The concurrence of OPN-positive expression and VM correlated with pCR $(P<0.001)$.

Angiogenesis is a key step in tumor growth and metastasis. OPN regulates hypoxia-inducible factor- $1 \alpha$-dependent VEGF expression, leading to breast tumor growth and angiogenesis in response to hypoxia. ${ }^{29} \mathrm{VM}$ is a microvascular channel made up of non-endothelial cells. It is a new model of neovascularization in aggressive tumors, caused by the specific capacity of malignant cells to form vessel-like networks, which provide sufficient blood supply for tumor growth. ${ }^{32}$ A previous study reported that VM patterns were always correlated with OPN expression. ${ }^{13}$ Another research indicated that OPN-positive expression played an important role in promoting the proliferation, tumorigenicity, and ability to form VM of spheroid-forming cells in breast cancer. ${ }^{21}$

In our study, OPN expression was also found to have a significant correlation with VM in breast cancer. The results showed that the improved rates of pCR after NACT correlated with the concurrence of OPN-positive expression and VM. As OPN and VM cause angiogenesis, chemotherapy can reach the specific cells more effectively and increase the drug concentration in the tumor microenvironment, thus improving the effective rate of NACT. Therefore, the concurrence of OPN expression and VM in breast cancer may result in a sensitive response to NACT.

\section{Conclusion}

Our results indicated that the concurrence of OPN-positive expression and VM can predict the $\mathrm{pCR}$ to $\mathrm{NACT}$ in breast cancer. The efficiency of NACT in certain patients can be easily predicted by detecting the expression of OPN and VM.

\section{Acknowledgments}

This work was supported by the National Natural Science Foundation of China (grant no 81201886). The funders had no role in the study design, data collection and analysis, decision to publish, or preparation of the manuscript.

\section{Disclosure}

The authors report no conflicts of interest in this work.

\section{References}

1. Tu M, Li Y, Zeng C, et al. MicroRNA-127-5p regulates osteopontin expression and osteopontin-mediated proliferation of human chondrocytes. Sci Rep. 2016;6:25032.

2. Weber GF. The metastasis gene osteopontin: a candidate target for cancer therapy. Biochim Biophys Acta. 2001;1552(2):61-85.

3. Ashkar S, Weber GF, Panoutsakopoulou V, et al. Eta-1 (osteopontin): an early component of type-1 (cell-mediated) immunity. Science. 2000; 287(5454):860-864.

4. Shi SM, Su ZB, Zhao JJ, et al. Increased osteopontin protein expression may be correlated with poor prognosis in non-small-cell lung cancer: a meta analysis. $J$ Cancer Res Ther. 2016;12(1):277-282.

5. Liaw L, Birk DE, Ballas CB, Whitsitt JS, Davidson JM, Hogan BL. Altered wound healing in mice lacking a functional osteopontin gene (spp1). J Clin Invest. 1998;101(7):1468-1478.

6. Singh K, DeVouge MW, Mukherjee BB. Physiological properties and differential glycosylation of phosphorylated and nonphosphorylated forms of osteopontin secreted by normal rat kidney cells. J Biol Chem. 1990;265(30):18696-18701.

7. Oldberg A, Franzen A, Heinegard D. Cloning and sequence analysis of rat bone sialoprotein (osteopontin) cDNA reveals an Arg-Gly-Asp cell-binding sequence. Proc Natl Acad Sci U S A. 1986;83(23): 8819-8823.

8. Kim JH, Skates SJ, Uede T, et al. Osteopontin as a potential diagnostic biomarker for ovarian cancer. JAMA. 2002;287(13):1671-1679.

9. Rittling SR, Chambers AF. Role of osteopontin in tumour progression. Br J Cancer. 2004;90(10):1877-1881.

10. Ortiz-Martinez F, Sanmartin E, Pomares-Navarro E, et al. Osteopontin regulates VEGFA and ICAM-1 mRNA expression in breast carcinoma. Am J Clin Pathol. 2015;143(6):812-822.

11. Lee SH, Park JW, Go DM, et al. Ablation of osteopontin suppresses $\mathrm{N}$-methyl-N-nitrosourea and Helicobacter pylori-induced gastric cancer development in mice. Carcinogenesis. 2015;36(12):1550-1560.

12. Zhao M, Liang F, Zhang B, Yan W, Zhang J. The impact of osteopontin on prognosis and clinicopathology of colorectal cancer patients: a systematic meta-analysis. Sci Rep. 2015;5:12713.

13. Kadkol SS, Lin AY, Barak V, et al. Osteopontin expression and serum levels in metastatic uveal melanoma: a pilot study. Invest Ophthalmol Vis Sci. 2006;47(3):802-806. 
14. Tuck AB, Chambers AF, Allan AL. Osteopontin overexpression in breast cancer: knowledge gained and possible implications for clinical management. J Cell Biochem. 2007;102(4):859-868.

15. Anborgh PH, Mutrie JC, Tuck AB, Chambers AF. Role of the metastasis-promoting protein osteopontin in the tumour microenvironment. J Cell Mol Med. 2010;14(8):2037-2044.

16. Allan AL, George R, Vantyghem SA, et al. Role of the integrin-binding protein osteopontin in lymphatic metastasis of breast cancer. Am J Pathol. 2006;169(1):233-246.

17. Tuck AB, Hota C, Chambers AF. Osteopontin (OPN)-induced increase in human mammary epithelial cell invasiveness is urokinase (uPA)dependent. Breast Cancer Res Treat. 2001;70(3):197-204.

18. Furger KA, Allan AL, Wilson SM, et al. Beta(3) integrin expression increases breast carcinoma cell responsiveness to the malignancyenhancing effects of osteopontin. Mol Cancer Res. 2003;1(11): $810-819$.

19. Liu W, Xu G, Ma J, et al. Osteopontin as a key mediator for vasculogenic mimicry in hepatocellular carcinoma. Tohoku J Exp Med. 2011;224(1). 29-39.

20. Shevde LA, Das S, Clark DW, Samant RS. Osteopontin: an effector and an effect of tumor metastasis. Curr Mol Med. 2010;10(1): 71-81.

21. Shevde LA, Metge BJ, Mitra A, et al. Spheroid-forming subpopulation of breast cancer cells demonstrates vasculogenic mimicry via hsa-miR299-5p regulated de novo expression of osteopontin. J Cell Mol Med. 2010;14(6B):1693-1706.

22. Chen Y, Chen K, Xiao X, et al. Pretreatment neutrophil-to-lymphocyte ratio is correlated with response to neoadjuvant chemotherapy as an independent prognostic indicator in breast cancer patients: a retrospective study. BMC Cancer. 2016;16:320.
23. Zhang GC, Zhang YF, Xu FP, et al. Axillary lymph node status, adjusted for pathologic complete response in breast and axilla after neoadjuvant chemotherapy, predicts differential disease-free survival in breast cancer. Curr Oncol. 2013;20(3):e180-e192.

24. Tavassoli FA. Lobular and ductal intraepithelial neoplasia. Pathologe. 2008;29(suppl 2):107-111.

25. Frenkel S, Barzel I, Levy J, et al. Demonstrating circulation in vasculogenic mimicry patterns of uveal melanoma by confocal indocyanine green angiography. Eye. 2008;22(7):948-952.

26. Zhang S, Zhang D, Sun B. Vasculogenic mimicry: current status and future prospects. Cancer Lett. 2007;254(2):157-164.

27. Baeten CI, Hillen F, Pauwels P, de Bruine AP, Baeten CG. Prognostic role of vasculogenic mimicry in colorectal cancer. Dis Colon Rectum. 2009;52(12):2028-2035.

28. Liu TJ, Sun BC, Zhao XL, et al. CD133+ cells with cancer stem cell characteristics associates with vasculogenic mimicry in triple-negative breast cancer. Oncogene. 2013;32(5):544-553.

29. Bandopadhyay M, Bulbule A, Butti R, et al. Osteopontin as a therapeutic target for cancer. Expert Opin Ther Targets. 2014;18(8): 883-895.

30. McFarland DC, Naikan J, Rozenblit M, Mandeli J, Bleiweiss I, Tiersten A. Changes in pathological complete response rates after neoadjuvant chemotherapy for breast carcinoma over five years. J Oncol. 2016; 2016:4324863.

31. Wang-Lopez Q, Chalabi N, Abrial C, et al. Can pathologic complete response ( $\mathrm{pCR}$ ) be used as a surrogate marker of survival after neoadjuvant therapy for breast cancer? Crit Rev Oncol Hematol. 2015;95(1): 88-104.

32. Zhang J, Qiao L, Liang N, et al. Vasculogenic mimicry and tumor metastasis. J BUON. 2016;21(3):533-541.
OncoTargets and Therapy

\section{Publish your work in this journal}

OncoTargets and Therapy is an international, peer-reviewed, open access journal focusing on the pathological basis of all cancers, potential targets for therapy and treatment protocols employed to improve the management of cancer patients. The journal also focuses on the impact of management programs and new therapeutic agents and protocols on

\section{Dovepress}

patient perspectives such as quality of life, adherence and satisfaction. The manuscript management system is completely online and includes a very quick and fair peer-review system, which is all easy to use. Visit http://www.dovepress.com/testimonials.php to read real quotes from published authors. 